\title{
MRI Assessment of Paediatric Developmental Delay
}

\author{
Konika Chaudhary ${ }^{1}$, Shivali Vaibhav Kashikar ${ }^{2}$, Rajasbala Pradeep Dhande ${ }^{3}$ \\ 1, 2, 3 Department of Radiodiagnosis, Datta Meghe Institute of Medical Sciences, \\ Sawangi, Wardha, Maharashtra, India.
}

\section{ABSTRACT}

\section{BACKGROUND}

Developmental delay is defined as a significant delay in the infant's developmental domains. Magnetic resonance imaging (MRI) is the best diagnostic tool to investigate such cases. Evaluation of a child with developmental delay is essential because it allows for early diagnosis and treatment and helps in counselling the parents regarding the outcome and to identify any possible risk of recurrence.

\section{METHODS}

The present study was conducted in AVBR Hospital, Sawangi, Wardha. This crosssectional descriptive study was conducted for magnetic resonance imaging assessment of brain in patients with developmental delay aged three months to 15 years. Males and females were both included in our study. The paediatrics department referred all patients with developmental delay or delayed milestones to the radiology department, where they underwent MRI brain with gadolinium contrast (Brivo MR 3551.5 Tesla). A total of 104 patients was included in the present study.

\section{RESULTS}

In our present study, distribution of the MRI findings based on structural morphology was 49 (47.12\%), 43 (41.35\%), 30 (28.85\%), 11 (10.58\%), 8 (7.69\%), and 3 (2.88 $\%)$ patients with abnormal findings in ventricles, corpus callosum, white matter, grey matter, cerebellum, brainstem respectively. Use of contrast was significant only in 4 (3.85\%) patients. Use of contrast was ineffective in 100 (96.15\%) patients, and the difference was statistically significant $(\mathrm{P}<0.001)$, stating that the use of contrast is ineffective in such patients.

\section{CONCLUSIONS}

MRI evaluation of the brain contributes to the diagnosis of aetiologies of developmental delay. Clinical diagnosis of the developmental delay should not be the only endpoint. MRI is the best investigation with a high yield in such developmental delay patients.

\section{KEY WORDS}

Developmental Delay, MRI Brain, Paediatric Patients
Corresponding Author: Dr. Konika Chaudhary, Department of Radiodiagnosis, Jawaharlal Nehru Medical College, Datte Meghe Institute of Medical Sciences, Sawangi, Wardha, Maharshtra 442001, India. E-mail: konika1303@gmail.com

DOI: $10.14260 / \mathrm{jemds} / 2021 / 105$

How to Cite This Article:

Chaudhary K, Kashikar SV, Dhande RP. MRI assessment of paediatric developmental delay. J Evolution Med Dent Sci 2021;10(08):479-483, DOI: 10.14260/jemds/2021/105

Submission 22-10-2020,

Peer Review 28-12-2020,

Acceptance 04-01-2021,

Published 22-02-2021.

Copyright (c) 2021 Konika Chaudhary et al. This is an open access article distributed under Creative Commons Attribution License [Attribution 4.0 International (CC BY 4.0)] 


\section{BACKGROUND}

Development is a continuous process that begins in utero and progresses until maturity. 1,2 This process involves structural and functional stages of progress or growth. Right from the foetal stage until a full-grown individual, a person goes through a lot of changes. Child as a toddler show their fine or gross motor skills which are followed by social skills, cognition, adapting to different modes of communications: languages / speeches. With all added attributes the person is considered mature and capable to cope as an individual. Growth and development go hand in hand which may be termed as maturating and myelination of the nervous system. ${ }^{3}$ When a child is not capable to perform certain activities at a certain stage of life, maybe a concern and often is termed as 'Developmental Delay'. Developmental delay is defined as a significant delay in one or more developmental domains of children. ${ }^{1}$ Evaluation of a child with developmental delay is essential as it allows for early diagnosis and treatment and helps in counselling parents regarding the outcome of their child and to identify any possible risk of recurrence. Various factors viz genetic, environmental, nutritional, and chronic diseases can have an adverse effect of delay in milestones, which can be evaluated using four domains of gross motor, fine motor, and social and language skills. All cases of delayed milestones should undergo neuroimaging as recommended by the American Academy of Neurology. Magnetic resonance imaging is more preferred, compared to computed tomography. ${ }^{4} \mathrm{MRI}$ is an essential tool to diagnose the cause of delayed milestones. MRI aids in visualising structural abnormality and aetiological causes of delayed development.1,2 The abnormal neuro-radiological findings were seen in $60-65 \%$ cases and the most common aetiology was traumatic / neurovascular aetiology. The most common cause is hypoxic-ischemic injury, ${ }^{1,2}$ whereas the most common structural abnormality is at the ventricular level. The second most crucial structure involved in the developmental delay is the corpus callosum (hypoplasia and agenesis). Contrastenhanced MRI studies in children, using gadolinium, have not been performed as the preferred mode of imaging. Therefore, they seem to have limited mention in the literature available today. Gadolinium utility in the evaluation of developmental delay and its significance is being presented in this study. In most developmental delay cases, an MRI brain contrast study is unhelpful as only few cases showed minor enhancement. ${ }^{5}$

With this background, the present research work aimed to determine the structural and the etiological cause of developmental delay in paediatric patients by doing MRI and MRI contrast of the brain and to analyse significance of MRI in diagnosing these cases.

\section{METHODS}

The present study duration is two years from 2018 to 2020 , conducted in AVBR Hospital, Sawangi, Wardha. This crosssectional descriptive study was conducted for MRI brain assessment in the developmental delay of patients aged between three months to 15 years. The paediatrics department referred all the developmental delay or delayed milestones patients to the radiology department, where they underwent an MRI brain with gadolinium contrast (Brivo MR 3551.5 Tesla). A total sample size of 104 patients was included in the study. The sample size was estimated with the help of an expert statistician. Neuroimaging of all patients was done after proper history and examination. This included history of delayed milestones, epilepsy, mental retardation, neurological deficit, birth history related to antenatal, intranatal, and postnatal events, maternal medical history, antenatal history of drug intake, alcohol / smoking exposure. Intranatal history included complications during delivery, presentation, birth weight, cry at birth, and APGAR score. Postnatal history included duration of postnatal hospital stay and neonatal intensive care unit (NICU) stay. Family history, immunisation history was also taken. All those patients who were fulfilling the inclusion criteria were enrolled in our present study. After proper explanation of the procedure and the purpose of the procedure, written informed consent was obtained from the parents. All the children were properly sedated before neuroimaging. Routine sequences were acquired: axial, coronal, and sagittal images of $\mathrm{T} 1$ and $\mathrm{T} 2$, fluid-attenuated inversion recovery (FLAIR), diffusion-weighted imaging (DWI), apparent diffusion coefficient (ADC), and post-contrast T1 weighted sequences. A contrast study was done to evaluate the effectiveness of contrast in children with developmental delay.

"Following Widjaja et al. protocol.1,2,3 structures were systematically evaluated -

1. Ventricles: Size and morphology.

2. Corpus callosum: Thickness and morphology.

3. Grey and white matter: The sulci and gyri of the grey matter are based on normal MR brain anatomy.

4. Basal ganglia: Morphology.

5. Brain stem: Morphology.

6. Cerebellum: Morphology."

To evaluate the use and significance of the intravenous administration of contrast media various questions were formulated "Did the contrast -

1. Aid the diagnosis?

2. Was there any noticeable enhancement of the lesion?

3. Did it serve in evaluating the aetiology of developmental delay?"

Accordingly, two groups were developed, the first group comprised of the effectiveness of contrast in the evaluation of developmental delay and the second group to show that the contrast is not effective in the evaluation of developmental delay. ${ }^{5}$

\section{Statistical Analysis}

Data were tabulated in the MS Excel sheet, and data analysis was done using SPSS, version 23.

\section{RESULTS}

In our present study of 104 patients, the distribution according to gender was 57 (54.81\%) males and 47 (45.19\%) females. The ratio was $1.2: 1$ between males and females. 38 patients $(36.54 \%)$ were in the age range of 3 months to 2 years, 66 patients $(63.46 \%)$ were in the age range of more than 2 years to 15 years. In our present study of 104 patients, the 
distribution of the study population according to the birth weight was, $68(65.38 \%)$ patients in less than 2500 gm group and $36(34.62 \%)$ patients in more than 2500 gm group. There were $68(65.38 \%)$ patients who were born preterm, and 36 (34.62\%) were born full term. Developmental delay was found common in preterm children.

\begin{tabular}{|cc|c|}
\hline Clinical Features & No. of Patients & Percentage \\
\hline $\begin{array}{c}\text { Isolated developmental delay } \\
\text { Developmental delay with other } \\
\text { features i.e. seizures, neurological } \\
\text { deficit, structural abnormality, } \\
\text { metabolic / genetic } \\
\text { Total }\end{array}$ & 41 & $39.42 \%$ \\
\hline Table 1. Distribution According to the Clinical Features \\
\hline
\end{tabular}

Table 1 shows distribution according to the clinical features of developmental delay. There were 41 (39.42\%) patients with isolated developmental delay whereas 63 (60.58 $\%)$ had developmental delay with other features i.e. seizures, neurological deficit etc.

The distribution of the paediatric patients according to the clinical features. There were 70 (67.30\%), 42 (40.38\%), 32 (30.77\%), 21 (20.19\%) and 10 (9.62\%) patients with clinical features of seizures and other neurological defects, motor (fine and gross) delay, language / speech delay, social / emotional delay and cognitive impairment respectively.

Out of 104 patients $41(39.42 \%)$ patients had normal MRI findings whereas 63 (60.58 \%) had abnormal MRI findings.

\begin{tabular}{|c|c|c|c|}
\hline \multicolumn{2}{|c|}{ Morphological Features } & Number & Percentage \\
\hline \multirow{2}{*}{ Ventricles } & Abnormal & 49 & 47.12 \\
\hline & Normal & 55 & 52.88 \\
\hline \multirow{2}{*}{ Corpus callosum } & Abnormal & 43 & 41.35 \\
\hline & Normal & 61 & 58.65 \\
\hline \multirow{2}{*}{ White matter } & Abnormal & 30 & 28.85 \\
\hline & Normal & 74 & 71.15 \\
\hline \multirow{2}{*}{ Grey matter } & Abnormal & 11 & 10.58 \\
\hline & Normal & 93 & 89.42 \\
\hline \multirow{2}{*}{ Cerebellum } & Abnormal & 8 & 7.69 \\
\hline & Normal & 96 & 92.31 \\
\hline \multirow{2}{*}{ Brainstem } & Abnormal & 3 & 2.88 \\
\hline & Normal & 101 & 97.12 \\
\hline \multicolumn{2}{|c|}{ Total } & 104 & 100 \\
\hline \multicolumn{4}{|c|}{$\begin{array}{l}\text { Table 2. Distribution of Cases Based on the MRI Findings } \\
\text { - Structural Morphology }\end{array}$} \\
\hline
\end{tabular}

Table 2 shows, distribution of the MRI finding based on structural morphology, there were 49 (47.12\%), 43 (41.35\%), $30(28.85 \%), 11(10.58 \%), 8(7.69 \%)$ and $3(2.88 \%)$ patients with abnormal MRI findings in ventricles, corpus callosum, white matter, grey matter, cerebellum, brainstem respectively.

It was also observed that there were $41(39.42 \%), 31$ (29.81\%), 24 (23.08\%), 6 (5.77\%) and 2 (1.92\%) patients in the etiological category of normal, traumatic / neurovascular, congenital / developmental, metabolic / degenerative and nonspecific respectively. The distribution of patients based on diagnosis. There were $31(49.20 \%)$ patients with hypoxic ischemic injury / encephalopathy and 5 (7.94\%) with isolated abnormality of corpus callosum.

There were $4(6.35 \%)$ patients each with aqueductal stenosis, tuberous sclerosis, Dandy-Walker malformation and hydrocephalus with thinning of corpus callosum respectively. Isolated hydrocephalus and Chiari malformation were seen in $2(3.17 \%)$ patients each. Alexander disease, metachromatic leukodystrophy, wide open schizencephaly, porencephalic cyst, lissencephaly pachygyria spectrum, polymicrogyria, hemimegaloencephaly and agenesis of cerebellum was observed in 1 (1.59\%) patient respectively.

\begin{tabular}{|cccc|}
\hline Effectiveness & Number of Patients & Percentage & P-Value \\
Effective & 4 & $3.85 \%$ & $\chi^{2}=88.615$ \\
Not effective & 100 & $96.15 \%$ & $\mathrm{P}<0.001^{* *}$ \\
Total & $\mathbf{1 0 4}$ & $\mathbf{1 0 0} \%$ & \\
\hline \multicolumn{3}{|c}{ Table 3. Effectiveness of Contrast } \\
\hline
\end{tabular}

Table 3 shows that the use of contrast was effective only in $4(3.85 \%)$ patients whereas ineffective in 100 (96.15\%) patients and the difference was statistically significant $(\mathrm{P}<$ 0.001) stating that use of contrast is ineffective in such patients.

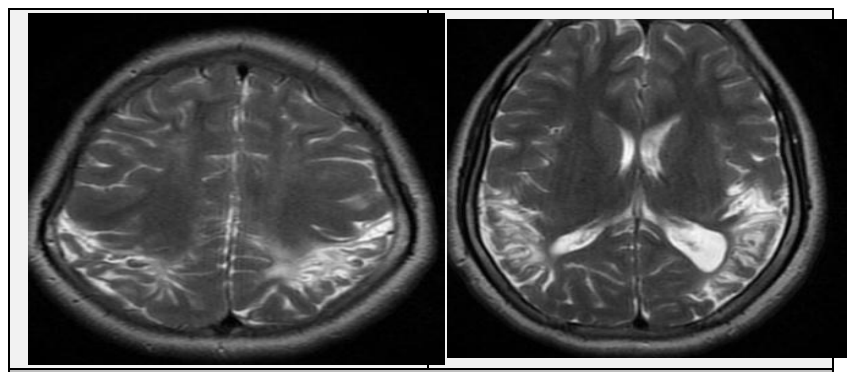

Figure 1. In a 6-Years-Old Child a) and b) Axial MRI Brain T2WI Shows Altered Signal Intensity in Bilateral Peri Trigonal White Matter Appearing Hyperintense with Atrophy of Overlying Cortex of Bilateral Occipito-Parietal Region. There is e / o Asymmetrical Mild Dilatation of the Posterior Horns of Bilateral Lateral Ventricles Suggestive of Hypoxic Ischaemic Encephalopathy.
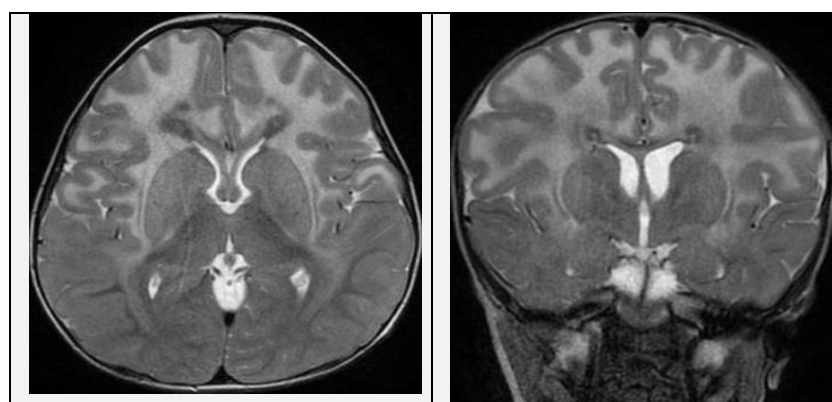

Figure 2. In a 3 Years Old Child a) Axial and b) Coronal T2 Weighted Images Shows Symmetrical Diffuse White Matter Hyperintensities Predominantly in the Frontal Lobes and Extending into the Parietal Lobes Suggestive of Possibility of LeucoDystrophy Most Likely to Be Alexander Disease.

\section{DISCUSSION}

Global developmental delay is slightly more common in male children. Developmental delay presents a broad spectrum of aetiologies, clinical findings, and MRI features ranging from utterly normal to abnormal. In our present study of 104 patients the distribution according to gender was 57 (54.81\%) males and 47 (45.19\%) females. The ratio was 1.2:1 between males and females. Thirty-eight patients (36.54\%) were in the age range of 3 months to 2 years, 66 patients (63.46\%) were in the age range of more than 2 years to 15 years. In our present study, distribution of the study population according to the birth weight was, $68(65.38 \%)$ patients in less than 2500 gm group and $36(34.62 \%)$ patients in more than 2500 gm. $68(65.38 \%)$ patients were born preterm and 36 (34.62 $\%)$ were born full term.

Rini Palve et al. ${ }^{1} 2016$ reported that 30 were males while 20 were female patients. Ali $\mathrm{AS}^{2} 2015$ said in his research, males (57 \%) were slightly more in number than the females 
(43\%), although there is no considerable difference. Jauhari et al. 20136, Shevell et al. 1999 also reported male gender preponderance in their studies for which no plausible mechanism has been proposed. ${ }^{7}$ Widjaja et al. reported that only " 22 patients were in group A (age less than 2 years), and 69 patients were in group B (more than 2 years - 18 years). Hence, most patients were in group B, similar to our study as the developmental delay is more noticeable during early school years. It is a symptom, neither a disease nor a diagnosis. ${ }^{3}$ Wong \& Chen et al. ${ }^{8}$ found low birth weight in only 96 of 537 children with a global developmental delay, which is less than ours (42.2\%). This may be because of their criterion, which these authors took as low birth weight was common in children less than $2 \mathrm{Kg}$ of body weight at birth".

In our current research, the developmental delay was found common in preterm children. Wong \& Chen et al. ${ }^{8}$ in his study, observed out of 537 children with global developmental delay, 96 (17.8\%) were born preterm. There were $70(67.30$ \%), 42 (40.38\%), 32 (30.77\%), 21 (20.19\%), and 10 (9.62\%) patients with clinical features of seizures and other neurological defects, motor (fine and gross)delay, language / speech delay, social / emotional delay, and cognitive impairment respectively. Distribution according to the clinical features of developmental delay, 41 (39.42\%) patients with isolated developmental delay, whereas 63 (60.58\%) had a developmental delay with other components, i.e., seizures and neurological deficits structural abnormality, metabolic / genetic. (Table 1)

Ali AS et al. 2015 reported "that out of 81 patients, isolated developmental delay was present in 8 patients, and developmental delay plus other clinical features such as seizures, neurological deficit, a structural abnormality was seen in 73 patients; hence, the majority of patients had developmental delay plus other symptoms".

In our study, 41 (39.42\%) patients had normal MRI findings, whereas 63 (60.58 \%) had abnormal MRI findings. Similar results were shown by Ali AS et al. ${ }^{2}$ they reported "that out of 81 children with developmental delay only $32 \%$ (26 cases) had normal MRI findings and they were advised further evaluation to diagnose the idiopathic cause of developmental delay. Abnormal findings were seen in remaining $68 \%$ (55 cases)"

In our research, distribution of the MRI finding based on structural morphology were 49 (47.12\%), 43 (41.35\%), 30 (28.85\%), $11(10.58 \%), 8$ (7.69\%), and 3 (2.88\%) patients with abnormal findings in ventricles, corpus callosum, white matter, grey matter, cerebellum, brainstem respectively. The majority of cases had a ventricular abnormality, followed by the corpus callosum. The majority of children with abnormal MRI findings were in a traumatic / neurovascular category, followed by congenital / developmental anomalies. Similar findings were seen in these studies. Rini Palve et al. ${ }^{1} 2016$ showed that corpus callosum abnormalities and ventricles were seen in 10 patients out of 42 patients.

There were 41 (39.42\%), 31 (29.81\%), 24 (23.08\%), 6 $(5.77 \%)$ and $2(1.92 \%)$ patients in the etiological category of normal, traumatic / neurovascular, congenital / developmental, metabolic / degenerative and nonspecific respectively. Majority of children with abnormal MRI findings were in traumatic / neurovascular category followed by congenital / developmental anomalies. Rini Palve et al. ${ }^{1}$ reported that "out of 42 patients there were 8 patients with traumatic / neurovascular diseases followed by congenital and developmental anomalies". Ali AS et al. ${ }^{2} 2015$ reported that "normal were $32 \%$ and abnormal findings of which traumatic / neurovascular diseases, congenital \& developmental, metabolic and degenerative, neoplastic and nonspecific were $31 \%, 17 \%, 10 \%, 2.5 \%$ and $7.5 \%$ respectively".

In our present study, distribution based on diagnosis showed that there were $31(49.20 \%)$ patients with hypoxicischemic injury / encephalopathy and 5 (7.94\%) with an isolated abnormality of the corpus callosum. There were 4 $(6.35 \%)$ patients with aqueductal stenosis, tuberous sclerosis, Dandy-Walker malformation, and hydrocephalus with thinning of the corpus callosum, respectively. Isolated hydrocephalus and Chiari malformation were seen in 2 (3.17 \%) patients each. Alexander disease, metachromatic leukodystrophy, wide-open schizencephaly, porencephalic cyst, lissencephaly pachygyria spectrum, polymicrogyria, hemimegaloencephaly, and agenesis of the cerebellum was observed in 1 (1.59\%) patient, respectively.

The majority of the patients were diagnosed with hypoxicischemic encephalopathy in our present study. Similar results were observed in Rini Palve et al. ${ }^{1}$ study.

We found the use of contrast was significant only in 4 (3.85 $\%)$ patients. It was ineffective in $100(96.15 \%)$ patients, and the difference was statistically significant $(\mathrm{P}<0.001)$, stating that the use of contrast is ineffective in such patients. Foerster BR et al. ${ }^{5}$ reported that gadolinium contrast does not yield good results in children with developmental delay.

However, MRI imaging is an integral part of the comprehensive evaluation of children with developmental delay. Many specific aetiological and pathophysiologic conditions that lead to developmental delay can be detected easily.

\section{CONCLUSIONS}

MRI evaluation of the brain contributes to the diagnosis of aetiologies of developmental delay. Clinical diagnosis of the developmental delay should not be the only endpoint. MRI is the best investigation with a high yield in such developmental delay patients.

Data sharing statement provided by the authors is available with the full text of this article at jemds.com.

Financial or other competing interests: None.

Disclosure forms provided by the authors are available with the full text of this article at jemds.com.

\section{REFERENCES}

[1] Palve R, Verma P, Chandnani S, et al. MRI evaluation of brain in children with developmental delay. International Journal of Contemporary Medical Research 2016;3(12):3461-3.

[2] Ali AS, Syed NP, Murthy GS, et al. Magnetic resonance imaging (MRI) evaluation of developmental delay in pediatric patients. J Clin Diagn Res 2015;9(1):TC21-4.

[3] Widjaja E, Nilsson D, Blaser S, et al. White matter abnormalities in children with idiopathic developmental delay. Acta Radiol 2008;49(5):589-95. 
[4] Shevell MI, Ashwal S, Donley D, et al. Practice parameter: evaluation of the child with global developmental delay: report of the quality standards subcommittee of the American academy of neurology and the practice committee of the child neurology society. Neurology 2003;60(3):367-80.

[5] Foerster BR, Ksar J, Petrou M, et al. Value of gadolinium in brain MRI examinations for developmental delay. Pediatric Neurology 2006;35(2):126-30.
[6] Jauhari P, Boggula R, Bhave A, et al. Aetiology of intellectual disability in paediatric outpatients in Northern India. Dev Med Child Neurol 2011;53(2):16772.

[7] Shevell MI, Majnemer A, Rosenbaum P, et al. Etiologic determination of childhood developmental delay. Brain Dev 2001;23(4):228-35.

[8] Chen IC, Chen CL, Wong MK, et al. Clinical analysis of 1048 children with developmental delay. Chang Gung Med J 2002;25(11):743-50. 